Pacific Journal of Mathematics

CONSTRUCTION OF A CLASS OF MODULAR FUNCTION 


\title{
CONSTRUCTION OF A CLASS OF MODULAR FUNCTIONS AND FORMS
}

\author{
MARVIN ISADORE KNOPP
}

1. Introduction. Let $G(j)$ be the principal congruence subgroup, of level $j$, of the modular group. In this paper we construct functions which are invariant under $G(j)$, for each integer $j \geqq 2$.

We begin by defining certain functions $\lambda_{\nu}(j ; \tau)$ which, although not in general invariant under $G(j)$, do possess the transformation properties

$$
\lambda_{\nu}(j ; T \tau)=\lambda_{\nu}(j ; \tau)+\text { constant, for all } T \text { in } G(j) .
$$

"This is the content of the main theorem, Theorem (4.02). Once this result has been established it is a simple matter to construct invariants for $G(j)$ by forming certain linear combinations of the $\lambda_{\nu}(j ; \tau)$. This is done in $\S 5$.

These functions $\lambda_{\nu}(j ; \tau)$ are defined as Fourier series which generalize the Fourier series expansion of $\lambda(\tau)$, given by Simons [6]. To derive the transformation equations (1.01), we proceed directly from the Fourier series, extending a method introduced by Rademacher [4], and since generalized by Lehner [2] and the author [1]. Although in [4] only the invariant $J(\tau)$ for the modular group is treated, the method of [4] has much wider applicability. Thus, in [2] it is used in the case of the modular group to overcome the usual convergence difficulties encountered in constructing forms of dimension -2 by means of Poincare series, while in [1] it is used to construct forms of nonnegative even integral dimension (in which case we, of course, do not have the method of the Poincaré series) for the modular group and several other closely related groups.

We will indicate in section 6 how the method of this paper can be used to construct automorphic forms of all positive even integral dimensions for the groups $G(j)$. In a future publication these same methods will be applied to construct automorphic functions and forms for certain other congruence subgroups of the modular group and for congruence subgroups of several other groups.

I would like to thank the referee of this paper for his helpful remarks.

2. Several lemmas. In [4] the principal analytic tool is a rather delicate lemma in which the terms of a certain conditionally convergent double series are rearranged. Several variations of this lemma can be

Received January 20, 1960 The author is a National Science Fellow. 
found in [1] and [2]. In this section we derive two generalizations of the lemma that will be needed in $\S 4$.

Lemma (2.01). Let $a<0, b<0, d>c>0$. Let $y>0, r \geqq 0$, and $\nu$ and $j$ be positive integers. Let $t=(c-1 / 2 b) d^{-1}$. Then

$$
\begin{aligned}
\sum_{k=1}^{\infty} \# \lim _{N \rightarrow \infty} \sum_{|m| \leqq N}^{*} \frac{e^{-2 \pi i \nu m^{\prime} \mid k}}{k^{1+r}(k i y-m)} \\
=\lim _{k \rightarrow \infty}\left\{\sum_{k=1}^{[K(a t-c)]} \sum_{k=-b k|a| \leqq K / a}^{*} \frac{e^{-2 \pi i \nu m^{\prime} \mid k}}{k^{1+r}(k i y-m)}\right. \\
\left.\quad+\sum_{k=[K(a t-c)]+1}^{[K(a t+c)]} \frac{a k-K t}{c} \sum_{\leqq m \leqq \frac{b k+K}{a}}^{*} \frac{e^{-2 \pi i v m^{\prime} \mid k}}{k^{1+r}(k i y-m)}\right\} .
\end{aligned}
$$

where the asterisk $\left(^{*}\right)$ indicates that the inner sum is taken on those $m$ such that $(m, k)=1$ and $m \equiv 1(\bmod j)$, the sharp (\#) indicates that the outer sum is taken on those $k$ such that $k \equiv j\left(\bmod j^{2}\right)$, and $m^{\prime}$ is is defined by $m m^{\prime} \equiv-1(\bmod k)$.

Lemma (2.03). Let $y, r, \nu$, and $j$ be as above. Let $\rho$ be any positive number. Then

$$
\begin{aligned}
\sum_{k=1}^{\infty} \lim _{N \rightarrow \infty} & \sum_{|m| \leqq N}^{*} \frac{e^{-2 \pi i \nu m^{\prime} / k}}{k^{1+r}(k i y-m)} \\
= & \lim _{K \rightarrow \infty} \sum_{k=1}^{[\rho K]} \sum_{|m| \leqq K}^{*} \frac{e^{-2 \pi i \nu m^{\prime} / k}}{k^{1+r}(k i y-m)} .
\end{aligned}
$$

REMARK. With care, (2.03) could have been included as a special case of (2.01). However, it is simpler and somewhat more germane to. our purpose to state them as separate lemmas. It should be noted that Lemma (2.03) is the same as a lemma in [1], except for the congruence conditions on $m$ and $k$.

A geometric interpretation may be helpful. By a "lattice point" we will mean a pair of relatively prime integers $k, m$ such that $k \equiv j\left(\bmod j^{2}\right)$ and $m \equiv 1(\bmod j)$. Rademacher's lemma [4] shows that the sum can be taken by first summing over the lattice points of the half square in the $k-m$ plane defined by $1 \leqq k \leqq K,|m| \leqq K$, and then letting $K \rightarrow \infty$. Lemma (2.03) allows us to first sum over the lattice points of the rectangle $1 \leqq k \leqq[\rho K],|m| \leqq K$, while Lemma. (2.01) shows that the sum can be taken first over the lattice points. of the trapezoid bounded by the lines $k=0, m=(a k-K t) / c, m=$ $(b k-K) / d, m=(b k+K) / d$.

The lemma can actually be proved for other trapezoids, but the form in which we have stated it will suffice for our application.

Proof of (2.01). We prove the lemma in the case $r=0$, the proof 
for $r>0$ being virtually the same. We first show the convergence of the left hand side of (2.02).

$$
\sum_{|m| \leqq N}^{*} \frac{e^{-2 \pi i m^{\prime} \nu / k}}{k(k i y-m)}=k^{-1} \sum_{0 \leqq h<k}^{*} e^{-2 \pi i h^{\prime} \nu / k} \sum_{\substack{n \\|n k+h| \leqq N}} \frac{1}{k i y-h-n k},
$$

where we have put $m=h+n k$. Therefore,

$$
\begin{aligned}
& \lim _{N \rightarrow \infty} \sum_{|m| \leqq N}^{*} \frac{e^{-2 \pi i m^{\prime} \nu / k}}{k(k i y-m)}=k^{-2} \sum_{0 \leqq h<k}^{*} e^{-2 \pi i h^{\prime} \nu / k} \lim _{N \rightarrow \infty} \sum_{\substack{n \\
|n k+h| \leqq N}} \frac{1}{i y-h / k-n} \\
& \quad=k^{-2} \sum_{0 \leqq h<k}^{*} e^{-2 \pi i h^{\prime} \nu / k} \cdot 2 \pi i\left(1 / 2-\left\{1-e^{2 \pi i(i y-h / k)}\right\}^{-1}\right) \\
& \quad=\pi i k^{-2} \sum_{0 \leqq h<k}^{*} e^{-2 \pi i h^{\prime} \nu / k}-2 \pi i k^{-2} \sum_{p=0}^{\infty} e^{-2 \pi y p} \sum_{0 \leqq h<k}^{*} \exp \left[-\frac{2 \pi i}{k}\left(\nu h^{\prime}+p h\right)\right] .
\end{aligned}
$$

Now, the inner sum of the second term is a Kloosterman sum, for which we have the estimate (see [5])

$$
\sum_{0 \leqq h<k}^{*} \exp \left[-\frac{2 \pi i}{k}\left(\nu h^{\prime}+p h\right)\right]=O\left(k^{2 / 3+\varepsilon}\right) .
$$

Also, the sum in the first term can be written

$$
\sum_{0 \leqq h<k}^{*} \exp \left[-\frac{2 \pi i}{k}\left(\nu h^{\prime}+k h\right)\right]=O\left(k^{2 / 3+\varepsilon}\right) .
$$

We conclude that

$$
\lim _{N \rightarrow \infty} \sum_{|m| \leqq N}^{*} \frac{e^{-2 \pi i m^{\prime} \nu / k}}{k(k i y-m)}=O\left(k^{-4 / 3+\varepsilon}\left\{1-e^{-2 \pi y}\right\}^{-1}\right), .
$$

and the left hand side of (2.01) converges.

Let $Z$ denote the set of integers. Let $z_{1}(K)=[K(d t-c)]$ and $z_{2}(K)=[K(d t+c)]$. We let $\mathscr{A}(K, N)=\{m \in Z \mid-N \leqq m<(b k-K) / d$ or $(b k+K) / d<m \leqq N\}$ and $\mathscr{B}(K, N)=\{m \in Z \mid(b k+K) / d<m \leqq N$ or $-N \leqq m<(a k-K t) / c\}$.

We can now state the lemma in the following form

$$
\begin{aligned}
\lim _{K \rightarrow \infty}\left\{\sum_{k=1}^{z_{1}(K)} \lim _{N \rightarrow \infty} \sum_{m \in \mathscr{A}(K, N)}^{*} \frac{e^{-2 \pi i m^{\prime} \nu / k}}{k(k i y-m)}\right. \\
\left.\quad+\sum_{k=z_{1}(K)+1}^{z_{2}(K)} \lim _{N \rightarrow \infty} \sum_{m \in \Re^{\prime}(K, N)}^{*} \frac{e^{-2 \pi i m^{\prime} \nu / k}}{k(k i y-m)}\right\}=0 .
\end{aligned}
$$

The function defined by

$$
g(m)=\left\{\begin{array}{l}
e^{-2 \pi i m^{\prime} \nu / k}, \text { if }(m, k)=1 \text { and } m \equiv 1(\bmod j) \\
0, \text { otherwise }
\end{array}\right.
$$

is periodic modulo $k$. This is easily seen if we recall that $k \equiv j\left(\bmod j^{2}\right)$ 
and therefore $j \mid k$. It follows that

where

$$
g(m)=\sum_{l=1}^{k} B_{l} e^{2 \pi i l m / k},
$$

$$
B_{l}=k^{-1} \sum_{0 \leqq m<k}^{*} \exp \left[-\frac{2 \pi i}{k}\left(\nu m^{\prime}+l m\right)\right] .
$$

Using (2.05) we see that

$$
B_{l}=O\left(k^{-1 / 3+\varepsilon}\right) \text {. }
$$

In the first double sum of (2.06) put

$$
\begin{aligned}
T_{k}(K, N) & =\sum_{m \in \mathscr{A}(K, N)}^{*} \frac{e^{-2 \pi i v m^{\prime} \mid k}}{k(k i y-m)}=\sum_{m \in \mathscr{A}(K, N)} \sum_{l=1}^{k} B_{l} \frac{e^{2 \pi i l m / k}}{k(k i y-m)} \\
& =\sum_{i=1}^{k-1} B_{l} \sum_{m \in \mathscr{A}(K, N)} \frac{e^{2 \pi i l m / k}}{k(k i y-m)}+B_{k} \sum_{m \in \mathscr{A}(K, N)} \frac{1}{k(k i y-m)} .
\end{aligned}
$$

Let $T_{k}(K)=\lim _{N \rightarrow \infty} T_{k}(K, N), z_{3}(K)=[(K+b k) / d]$, and $z_{4}(K)=[(K-b k) / d]$. Recalling the definition of $\mathscr{A}(K, N)$ and making use of (2.08), we may; write

$$
\begin{aligned}
T_{k}(K)= & k^{-1} \sum_{i=1}^{k-1} B_{l} \sum_{m=z_{3}(K)+1}^{\infty} \frac{e^{2 \pi i l m / k}}{k i y-m} \\
& +k^{-1} \sum_{l=1}^{k-1} B_{l} \sum_{m=z_{4}(K)+1}^{\infty} \frac{e^{-2 \pi i l m / k}}{k i y+m} \\
& +B_{k} k^{-1} \sum_{m=z_{4}(K)+1}^{\infty}\left(\frac{1}{k i y-m}+\frac{1}{k i y+m}\right) \\
& +B_{k} k^{-1} \sum_{m=z_{3}(K)+1}^{z_{4}(K)} \frac{1}{k i y-m} \\
= & S_{1}+S_{2}+S_{3}+S_{4} .
\end{aligned}
$$

To handle $S_{1}$, put

$$
E_{m}=\sum_{p=z_{3}(\mathbb{K})+1}^{m} e^{2 \pi i l p / k}=\frac{e^{\pi i l(2 m+1) / k}-e^{\pi i l\left(2 z_{3}(K)+1\right) / k}}{e^{\pi i l / k}-e^{-\pi i l / k}} .
$$

Therefore,

$$
\left|E_{m}\right| \leqq(\sin \pi l / k)^{-1} \leqq(\min \{2 l / k, 2(k-l) / k\})^{-1} \leqq \frac{k}{2}(1 / l+1 /(k-l))
$$

Now,

$$
\begin{aligned}
\sum_{m=z_{3}(K)+1}^{\infty} \frac{e^{2 \pi i l m / k}}{k i y-m} & =\sum_{m=z_{3}(\mathrm{~K})+1}^{\infty} \frac{E_{m}-E_{m-1}}{k i y-m} \\
& =\sum_{m=z_{3}(K)+1}^{\infty} E_{m}\left(\frac{1}{k i y-m}-\frac{1}{k i y-m-1}\right) .
\end{aligned}
$$


Hence,

$$
\begin{aligned}
& \left|\sum_{m=z_{3}(K)+1}^{\infty} \frac{e^{2 \pi i l m / k}}{k i y-m}\right| \\
& \quad \leqq \frac{k}{2}(1 / l+1 /(k-l)) \sum_{m=z_{3}(K)+1}^{\infty}\left\{k^{2} y^{2}+m^{2}\right\}^{-1 / 2}\left\{k^{2} y^{2}+(m+1)^{2}\right\}^{-1 / 2} \\
& \quad<\frac{k}{2}(1 / l+1 /(k-l)) \int_{z_{3}(K)}^{\infty} \frac{d x}{x^{2}}=\frac{k}{2}(1 / l+1 /(k-l))[(K+b k) / d]^{-1} .
\end{aligned}
$$

Now, we are here considering only those $k$ in the range $1 \leqq k \leqq z_{1}(K)$ $=[K(d t-c)] . \quad$ Since $b<0, d>0,(K+b k) / d \geqq\{K+K b(d t-c)\} / d=$ $K / 2 d$. Making use of (2.07), we conclude that

$$
\begin{aligned}
S_{1} & =k^{-1} \sum_{l=1}^{k-1} B_{l} \sum_{m=z_{3}(K)+1}^{\infty} \frac{e^{2 \pi i l m / k}}{k i y-m} \\
& =O\left(k^{-1} \sum_{l=1}^{k-1} k^{-1 / 3+\varepsilon} \frac{k}{2}\{1 / l+1 /(k-l)\} K^{-1}\right) .
\end{aligned}
$$

Therefore,

$$
S_{1}=O\left(k^{-1 / 3+\varepsilon} K^{-1} \log k\right) .
$$

We can estimate $S_{2}$ in exactly the same way simply by noticing that $(K-b k) / d \geqq K / d$. We obtain

$$
S_{2}=O\left(k^{-1 / 3+\varepsilon} K^{-1} \log k\right) .
$$

The estimation of $S_{3}$ is simpler. We notice that

$$
S_{3}=B_{k} \cdot k^{-1} \sum_{m=z_{4}(k)+1}^{\infty} \frac{2 i y k}{-y^{2} k^{2}-m^{2}}
$$

and hence

$$
\left|S_{3}\right|<\left|B_{k}\right| \sum_{m=z_{4}(K)+1}^{\infty} \frac{2 y}{m^{2}}<\left|B_{k}\right| \int_{z_{4}(K)}^{\infty} \frac{2 y d x}{x^{2}} .
$$

Therefore,

$$
S_{3}=O\left(k^{-1 / 3+\varepsilon}[(K-b k) / d]^{-1}\right)=O\left(k^{-1 / 3+\varepsilon} K^{-1}\right) .
$$

We consider $S_{4}$. Recalling that $z_{3}(K)+1>(K+b k) / d \geqq K / 2 d$, we find that

$$
\begin{aligned}
\left|\sum_{m=z_{3}(K)+1}^{z_{4}(K)} \frac{1}{k i y-m}\right| & \leqq \sum_{m=z_{3}(K)+1}^{z_{4}(K)}\left(k^{2} y^{2}+m^{2}\right)^{-1 / 2} \leqq \sum_{m=z_{3}(K)+1}^{z_{4}(K)}\left(k^{2} y^{2}+K^{2} / 4 d^{2}\right)^{-1 / 2} \\
& \leqq 2 d K^{-1}\{(K-b k) / d-(K+b k) / d\}=-4 b k K^{-1}
\end{aligned}
$$

Therefore, using (2.07), 


$$
S_{4}=B_{k} \cdot k^{-1} \sum_{m=z_{3}(K)+1}^{z_{4}(K)} \frac{1}{k i y-m}=O\left(k^{-1 / 3+\varepsilon} K^{-1}\right)
$$

Collecting our results (2.10), (2.11), and (2.12), we have $T_{k}(K)=$ $O\left(k^{-1 / 3+\varepsilon} K^{-1} \log k\right)$. Hence,

$$
\begin{aligned}
\sum_{k=1}^{z_{1}(K)} \lim _{N \rightarrow \infty} \sum_{m \in \mathscr{A}(K, N)}^{*} \frac{e^{-2 \pi i \nu m^{\prime} / k}}{k(k i y-m)} & =\sum_{k=1}^{z_{1}(K)} T_{k}(K) \\
& =O\left(K^{-1} \sum_{k=1}^{z_{1}(K)} k^{-1 / 3+\varepsilon} \log k\right) \\
& =O\left(K^{-1 / 3+\varepsilon} \log K\right)
\end{aligned}
$$

In the second double sum of (2.06) put

$$
\begin{aligned}
U_{k}(K, N) & =\sum_{m \in \mathscr{O}(K, N)}^{*} \frac{e^{-2 \pi i \nu m^{\prime} / k}}{k(k i y-m)} \\
& =\sum_{i=1}^{k-1} B_{l} \sum_{m \in \mathscr{O}(K, N)} \frac{e^{-2 \pi i \nu m^{\prime} / k}}{k(k i y-m)}+B_{k} \sum_{m \in \mathscr{F}^{\prime}(K, N)} \frac{1}{k(k i y-m)} .
\end{aligned}
$$

Let $U_{k}(K)=\lim _{N \rightarrow \infty} U_{k}(K, N)$ and $z_{5}(K)=[(K t-a k) / c]$. Then using (2.15) and the definition of $\mathscr{B}(K, N)$ we find

$$
\begin{aligned}
U_{k}(K)= & k^{-1} \sum_{l=1}^{k-1} B_{l} \sum_{m=z_{5}(K)+1}^{\infty} \frac{e^{2 \pi i l m / k}}{k i y-m}+k^{-1} \sum_{l=1}^{k-1} B_{l} \sum_{m=z_{5}(K)+1}^{\infty} \frac{e^{-2 \pi i l m / k}}{k i y+m} \\
& +B_{k} \cdot k^{-1} \sum_{m=z_{5}(K)+1}^{\infty}\left(\frac{1}{k i y-m}+\frac{1}{k i y+m}\right) \\
& +k^{-1} \sum_{i=1}^{k-1} B_{l} \sum_{m=z_{3}(K)+1}^{z_{5}(K)} \frac{e^{-2 \pi i l m / k}}{k i y-m}+B_{k} \cdot k^{-1} \sum_{m=z_{3}(K)+1}^{z_{5}(K)} \frac{1}{k i y-m} \\
= & S_{5}+S_{6}+S_{7}+S_{8}+S_{9} .
\end{aligned}
$$

Since $(K t-a k) / c>K t / c$, we can estimate $S_{5}$ and $S_{6}$ in the same way as $S_{1}$, and $S_{7}$ in the same way as $S_{3}$ We obtain

$$
S_{5}+S_{6}+S_{7}=O\left(k^{-1 / 3+\varepsilon} K^{-1} \log k\right) .
$$

To handle $S_{8}$ define $E_{m}$ as before. Then

$$
\begin{aligned}
& \sum_{m=z_{3}(K)+1}^{z_{5}(K)} \frac{e^{2 \pi i l m / k}}{k i y-m} \\
& \quad=\sum_{m=z_{3}(K)+1}^{z_{5}(K)} E_{m}\left(\frac{1}{k i y-m}-\frac{1}{k i y-m-1}\right)+E_{z_{5}(K)} /\left(k i y-z_{5}(K)-1\right) .
\end{aligned}
$$

Recalling that $\left|E_{m}\right| \leqq(k / 2)\{1 / l+1 /(k-l)\}$, we have 


$$
\begin{aligned}
& \left|\sum_{m=z_{3}(K)+1}^{z_{5}(K)} \frac{e^{2 \pi i l m / k}}{k i y-m}\right| \\
& \leqq \frac{k}{2}\{1 / l+1 /(k-l)\}\left(\sum_{m=z_{3}(K)+1}^{z_{5}(K)}\left\{k^{2} y^{2}+m^{2}\right\}^{-1 / 2}\left\{k^{2} y^{2}+(m+1)^{2}\right\}^{-1 / 2}\right. \\
& \left.+\left\{k^{2} y^{2}+(K t-a k)^{2} / c^{2}\right\}\right) \\
& <\frac{k}{2}\{1 / l+1 /(k-l)\}\left(\sum_{m=z_{3}(K)+1}^{z_{5}(K)}\left(k^{-2} y^{-2}\right)+c / t K\right) \\
& \left.\leqq \frac{k}{2}\{1 / l+1 /(k-l)\}\left\{y^{-2} k^{-2}((K t-a k) / c-(K+b k) / d)\right)+c / t K\right\} \\
& \leqq \frac{k}{2}\{1 / l+1 /(k-l)\}\left(K y^{-2} k^{-2} c^{-1} d^{-1}\{(d t-c)+(-a d-b c)(d t+c)\}+c / t K\right),
\end{aligned}
$$

since $-a d-b c>0$ and $k$ is in the range $K(d t-c)<z_{1}(K)+1 \leqq k \leqq$ $z_{2}(K) \leqq K(d t+c)$. Therefore,

$$
\begin{aligned}
S_{8} & =k^{-1} \sum_{l=1}^{k-1} B_{l} \sum_{m=z_{3}(K)+1}^{z_{5}(K)} \frac{e^{2 \pi i l m / \hbar}}{k i y-m} \\
& =O\left(k^{-1} \sum_{l=1}^{k-1} k^{-1 / 3+\varepsilon} \cdot \frac{k}{2}\{1 / l+1 /(k-l)\}\left\{K k^{-2}+K^{-1}\right\}\right. \\
& =O\left(k^{-1 / 3+\varepsilon} \log k\left\{K k^{-2}+K^{-1}\right\}\right) .
\end{aligned}
$$

Finally, we estimate $S_{9}$.

$$
\begin{aligned}
&\left|\sum_{m=z_{3}(K)+1}^{z_{5}(K)} \frac{1}{k i y-m}\right| \leqq \sum_{m=z_{3}(K)+1}^{z_{5}(K)}\left(k^{2} y^{2}+m^{2}\right)^{-1 / 2} \leqq y^{-1} k^{-1}\{(K t-a k) / c-(K-b k) / d\} \\
& \leqq K(c d y k)^{-1}\{(d t-c)-(a b+b c)(d t+c)\} .
\end{aligned}
$$

Therefore,

$$
S_{9}=B_{k} \cdot k^{-1} \sum_{m=z_{3}(K)+1}^{z_{5}(K)} \frac{1}{k i y-m}=O\left(k^{-7 / 3+\varepsilon} K\right) .
$$

Using (2.17), (2.18), and (2.19), we find that

$$
U_{k}(K)=O\left(k^{-1 / 3+\varepsilon} \log k\left\{K^{-1}+K \cdot k^{-2}\right\}\right) .
$$

Hence,

$$
\begin{aligned}
& \sum_{k=z_{1}(K)+1}^{z_{2}(K)} \lim _{N \rightarrow \infty} \sum_{m \in \mathscr{B}(K, N)}^{*} \frac{e^{-2 \pi i \nu m^{\prime} \mid k}}{k(k i y-m)} \\
& \quad=\sum_{k=z_{1}(K)+1}^{z_{2}(K)} U_{k}(K)=O\left(\sum_{k=z_{1}(K)+1}^{z_{2}(K)} k^{-1 / 3+\varepsilon} \log k\left\{K^{-1}+K \cdot k^{-2}\right\}\right) \\
& \quad=O\left(K^{-1} \log K \sum_{k=z_{1}(K)+1}^{z_{2}(K)} k^{-1 / 3+\varepsilon}\right)=O\left(K^{-1} \log K \cdot K^{-1 / 3 \mid \varepsilon} \cdot 2 c K\right) \\
& \quad=O\left(K^{-1 / 3+\varepsilon} \log K\right) .
\end{aligned}
$$

Now (2.06) follows from (2.14) and (2.20) and the lemma is proved. 
Proof of (2.03). We outline the proof for the case $r=0$. The left hand side of (2.04) is the same as the left hand side of (2.02) and its convergence has already been demonstrated.

The lemma may be stated as follows

$$
\lim _{K \rightarrow \infty} \sum_{k=1}^{[\rho E]} \lim _{N \rightarrow \infty} \sum_{K<|m| \leqq N}^{*} \frac{e^{-2 \pi i \nu m^{\prime} \mid k}}{k(k i y-m)}=0 .
$$

Let

$$
\begin{aligned}
V_{k}(K, N) & =\sum_{K<|m| \leqq N}^{*} \frac{e^{-2 \pi i \nu m^{\prime} \mid k}}{k(k i y-m)} \\
& =\sum_{l=1}^{k-1} B_{l} \sum_{K<|m| \leqq N}^{*} \frac{e^{2 \pi i l m / k}}{k(k i y-m)}+B_{k} \sum_{K<|m| \leqq N} \frac{1}{k(k i y-m)} .
\end{aligned}
$$

Then,

$$
\begin{aligned}
V_{k}(K)= & \lim _{N \rightarrow \infty} V_{k}(K, N)=k^{-1} \sum_{l=1}^{k-1} B_{l} \sum_{m=k+1}^{\infty} \frac{e^{2 \pi i l m / k}}{k i y-m} \\
& +k^{-1} \sum_{l=1}^{k-1} B_{l} \sum_{m=K+1}^{\infty} \frac{e^{-2 \pi i l m / k}}{k i y+m}+B_{k} \cdot k^{-1} \sum_{m=K+1}^{\infty}\left(\frac{1}{k i y-m}+\frac{1}{k i y+m}\right) \\
= & S_{1}^{\prime}+S_{2}^{\prime}+S_{3}^{\prime} .
\end{aligned}
$$

Now $S_{1}^{\prime}$, and $S_{2}^{\prime}$ can be estimated in the same way as $S_{1}$ and $S_{3}^{\prime}$ in the same way as $S_{3}$. Once we have these estimates the proof of (2.21), proceeds exactly as the proof of (2.14) of the previous lemma.

3. The functions $\lambda_{\nu}(j ; \tau)$. Let $j$ be an integer $\geqq 2$ and let $\nu$ be a positive integer. We define the function

$$
\begin{aligned}
& \lambda_{\nu}(j ; \tau)=\sum_{n=1}^{\infty} a_{n}(\nu, j) e^{2 \pi i n \tau / j} \\
& a_{n}(\nu, j)=(\pi / 8) \sum_{k=1}^{\infty} k^{-1} A_{k, \nu}(n) \cdot(\nu / n)^{1 / 2} I_{1}\left(4 \pi(n \nu)^{1 / 2} / k\right),
\end{aligned}
$$

where

$$
A_{k, \nu}(n)=\sum_{0 \leqq h<k}^{*} \exp \left[\frac{-2 \pi i}{k}\left(\nu h^{\prime}+n h\right)\right],
$$

a Kloosterman sum, and $I_{1}$ is the modified Bessel function of the first kind. Recall that the sharp $\left(^{*}\right)$ means that we allow only those $k$ such that $k \equiv j\left(\bmod j^{2}\right)$ and the asterisk $\left(^{*}\right)$ indicates that we allow only those $h$ such that $h \equiv 1(\bmod j)$ and $(h, k)=1$.

We need the following

LEMMA (3.02). (a) If $a_{n}(\nu, j)$ is defined as in (3.01) then

$$
a_{n}(\nu, j) \sim\left\{\nu^{1 / 4} n^{-3 / 4}(2 j)^{-1 / 2} / 16\right\} e^{-2 \pi i(n-\nu) / s} \exp \left(4 \pi(n \nu)^{1 / 2} / j\right) .
$$


(b) If $|z|<1$, then

$$
\sum_{n=1}^{\infty} z^{n} \sum_{p=0}^{\infty}\left(4 \pi^{2} n \nu k^{-2}\right)^{p} / p !(p+1) !
$$

is absolutely convergent.

Proof. (a) The first term that occurs in the sum defining $a_{n}(\nu, j)$ is for $k=j$. This term is equal to

$$
(\pi / 8) j^{-1} A_{j, \nu}(n)(\nu / n)^{1 / 2} I_{1}\left(4 \pi(n \nu)^{1 / 2} / j\right) \text {. }
$$

But

$$
A_{\jmath, \nu}(n)=\exp [-2 \pi i\{n+(j-1) \nu\} / j]=e^{-2 \pi i(n-\nu) / j} .
$$

Therefore the first term is

$$
(\pi / 8) j^{-1} e^{-2 \pi i(n-\nu) / j} \cdot(\nu / n)^{1 / 2} I_{1}\left(4 \pi(n \nu)^{1 / 2} / j\right) .
$$

It follows that

$$
\begin{aligned}
\mid a_{n}(\nu, j)- & (\pi / 8 j) e^{-2 \pi i(n-\nu / j}(\nu / n)^{1 / 2} I_{1}\left(4 \pi(n \nu)^{1 / 2} / j\right) \mid \\
& =\left|(\pi / 8) \sum_{k=2 j}^{\infty} k^{-1} A_{k, \nu}(n)(\nu / n)^{1 / 2} I_{1}\left(4 \pi(n \nu)^{1 / 2} / k\right)\right| \\
& \leqq C_{1}(\nu / n)^{1 / 2} \sum_{k=2 j}^{\infty} k^{-1} k^{2 / 3+\varepsilon} I_{1}\left(4 \pi(n \nu)^{1 / 2} / k\right),
\end{aligned}
$$

where we have made use of (2.05)

It is a simple consequence of the power series definition of $I_{1}$

$$
I_{1}(\eta)=\sum_{p=0}^{\infty}(\eta / 2)^{2 p+1} / p !(p+1) !
$$

that $I_{1}(\eta) \leqq \sinh \eta$. We also need the fact that $\sinh \eta \leqq(\eta / B) \sinh B$, for $0 \leqq \eta \leqq B$. We find that

$$
\begin{aligned}
& \left|a_{n}(\nu, j)-(\pi / 8 j) e^{-2 \pi i(n-\nu) / j}(\nu / n)^{1 / 2} I_{1}\left(4 \pi(n \nu)^{1 / 2} / j\right)\right| \\
& \quad \leqq C_{1}(\nu / n)^{1 / 2} \sum_{k=2 j}^{\infty} k^{-1 / 3+\varepsilon}\left\{\left(4 \pi(n \nu)^{1 / 2} / k\right) /\left(4 \pi(n \nu)^{1 / 2} / 2 j\right)\right\} \sinh \left(4 \pi(n \nu)^{1 / 2} / 2 j\right) \\
& \quad \leqq C_{2} \exp \left(2 \pi(n \nu)^{1 / 2} / j\right) \cdot n^{-1 / 2} .
\end{aligned}
$$

Now in ([7], p. 203, formula 2), it is shown that $I_{1}(\eta) \sim e^{\eta} /(2 \pi \eta)^{1 / 2}$. Therefore,

$$
I_{1}\left(4 \pi(n \nu)^{1 / 2} / j\right) \sim \exp \left(4 \pi(n \nu)^{1 / 2} / j\right) / 2 \pi\left(2 j^{-1}\right)^{1 / 2}(n \nu)^{1 / 4} .
$$

and the result follows.

$$
\left.\sum_{p=0}^{\infty}\left(4 \pi^{2} n \nu / k^{2}\right)^{p} / p !(p+1) !=\left\{k / 2 \pi(n \nu)^{1 / 2}\right\} I_{\mathrm{i}}(4 \pi(n\lrcorner)^{1 / 2} / k\right)
$$




$$
\begin{aligned}
& \leqq\left\{k / 2 \pi(n \nu)^{1 / 2}\right\} \sinh \left(4 \pi(n \nu)^{1 / 2} / k\right) \\
& <\left\{k / 2 \pi(n \nu)^{1 / 2}\right\} \exp \left(4 \pi(n \nu)^{1 / 2} / k\right) .
\end{aligned}
$$

The result follows.

Lemma (302a) shows that the series defining $\lambda_{\nu}(j ; \tau)$ converges absolutely for $\mathscr{J}(\tau)>0$. Therefore, $\lambda_{\nu}(j ; \tau)$ is analytic in the upper half $\tau$-plane.

In order to derive the transformation properties of $\lambda_{\nu}(j ; \tau)$ we transform (3.01) into a certain double series. The computations involved are a repetition of those found in [4, pp. 244-5] and in [1] and [2] and we omit them. Briefly, the series definition of $a_{n}(\nu, j)$ is inserted into the series for $\lambda_{\nu}(j ; \tau), I_{1}$ is replaced by the power series (3.03), Lemma (3.02) is used to justify several interchanges of summation, and use is made of the Lipschitz formula [3]

$$
\begin{aligned}
& \sum_{n=1}^{\infty} n^{p}\{\exp [2 \pi i(\tau / j-h / k)]\}^{n} \\
&= \begin{cases}\left(p ! /(2 \pi)^{p+1}\right) \cdot \sum_{l=\infty}^{\infty}(-i \tau / j+i h / k+l i)^{-p-1}, & \text { for } p>0 \\
-1 / 2+(1 / 2 \pi) \lim _{N \rightarrow \infty} \sum_{l=-N}^{N}(-i \tau / j+i h / k+l i)^{-1}, & \text { for } p=0 .\end{cases}
\end{aligned}
$$

We obtain the double series

(3.04) $\quad \lambda_{\nu}(j ; \tau)=$ constant $+\frac{1}{16} \sum_{k=1}^{\infty} \sum_{0 \leq h<k}^{*} e^{-2 \pi i \nu h^{\prime} / \hbar}$

$$
\cdot \lim _{N \rightarrow \infty} \sum_{l=N}^{N}\left\{\exp \left[\frac{2 \pi i \nu}{k(k \tau / j-h-k l)}\right]-1\right\} .
$$

4. Transformation properties of the $\lambda_{\nu}(j ; \tau)$. In (3.04) put $m=$ $h+k l$. Since $j \mid k$ it follows that $m \equiv h(\bmod j)$. Hence $m \equiv 1(\bmod j)$ is a consequence of $h \equiv 1(\bmod j)$. Also $(h, k)=1 \mathrm{implies}(m, k)=1$. It is easy to see that as $l$ runs through all the integers and $h$ through a residue class modulo $k$ with the restrictions $(h, k)=1$ and $h \equiv 1(\bmod j)$, then $h+k l$ takes on, exactly once, each integer value $m$ such that $(m, k)=1$ and $m \equiv 1(\bmod j)$. Then $(3.04)$ becomes

$$
\lambda_{\nu}(j ; \tau)=A+\frac{1}{16} \sum_{k=1}^{\infty} \lim _{N \rightarrow \infty} \sum_{|m| \leqq N}^{*} e^{-2 \pi i \nu m^{\prime} \mid k}\left\{\exp \left[\frac{2 \pi i \nu}{k(k \tau / j-m)}\right]-1\right\} .
$$

Let $a, b, c, d$ be integers such that $a d-b c=1, a \equiv d \equiv 1(\bmod j)$, and $b \equiv c \equiv 0(\bmod j)$. Denote by $T_{a, b, c, a}$ the element of $G(j)$ defined by

$$
T_{a, b, c, a}(\tau)=\frac{a \tau+b}{c \tau+d}
$$

We wish to prove

THEOREM $(4.02)^{1}$. The function $\lambda_{\nu}(j ; \tau)$ satisfies the transformation

1 See correction at end of paper. 
equations

$$
\lambda_{\nu}\left(j ; T_{a, b, c, d}(\tau)\right) \equiv \lambda_{\nu}\left(j ; \frac{a \tau+b}{c \tau+d}\right)=\lambda_{\nu}(j ; \tau)+\omega_{\nu}(j ; c, d),
$$

for all $T_{a, b, c . d}$ in $G(j)$ and. $\mathscr{F}(\tau)>0$. Here $\omega_{\nu}(j ; c, d)$ does not depend on $\tau, a$, or $b$.

Proof. Let us suppose we have already shown that

$$
\lambda_{\nu}\left(j ; \frac{a \tau+b}{c \tau+d}\right)=\lambda_{\nu}(j ; \tau)+\omega,
$$

where $\omega$ does not depend on $\tau$. Under this assumption we prove that $\omega$ is independent of $a$ and $b$.

Let $T_{a^{\prime}, b^{\prime}, c, a}$ be in $G(j)$. Then, since $a-a^{\prime} \equiv b-b^{\prime} \equiv 0(\bmod j)$ and $a d-b c=a^{\prime} d-b^{\prime} c=1$, we have that $a^{\prime}=a+q^{\prime} j, b^{\prime}=b+r^{\prime} j$, with $q^{\prime}, r^{\prime}$ integers and $q^{\prime} d=r^{\prime} c$. Since $(c, d)=1$ it follows that $q^{\prime}=q c$, $r^{\prime}=q d$ with $q$ an integer, and therefore $a^{\prime}=a+q c j, b^{\prime}=b+q d j$. Hence $T_{a^{\prime}, b^{\prime}, c, d}=T_{1, q j, 0,1} \cdot T_{a, b, c, d}$, and clearly

$$
\lambda_{\nu}\left(j ; \frac{a^{\prime} \tau+b^{\prime}}{c \tau+d}\right)=\lambda_{\nu}\left(j ; \frac{a \tau+b}{c \tau+d}\right)=\lambda_{\nu}(j ; \tau)+\omega .
$$

Therefore, $\omega$ does not depend on $a$ or $b$.

It suffices to prove (4.03) subject to the restrictions $d>j c>0, a<0$, $b<0$. First we may assume $c>0$, changing the signs of $a, b, c, d$ if necessary. It is then simple to compute that $T_{a, b, c, a}=T_{1, s j, 0,1} \cdot T_{a, \beta, \gamma, \delta} \cdot T_{1,-r j, 0,1}$, with $\alpha=a-s j c, \beta=r j(\alpha-s j c)+b-s j d, \gamma=c, \delta=d+r j c$, and we can choose integers $r$ and $s$ so large that $\alpha<0, \beta<0, \delta>j c$. But $\lambda_{\nu}(j ; \tau)$ is clearly invariant under $T_{1, s j .0,1}$ and $T_{1,-r j, 0,1}$ since these are translations by $s j$ and $-r j$ respectively. Hence, if $\lambda_{\nu}\left(j ; T_{\alpha, \beta, \gamma, \delta}(\tau)\right)=$ $\lambda_{\nu}(j ; \tau)+\omega$, then $\lambda_{\nu}\left(j ; T_{a, b, c, a}(\tau)\right)=\lambda_{\nu}(j ; \tau)+\omega$.

Now, in order to apply Lemmas (2.01) and (2.03) we assume that $\tau$ is a pure imaginary number. Expanding the expression in the braces in (4.01) into a power series, we get

$$
\begin{aligned}
\lambda_{\nu}(j ; \tau)= & A+\frac{1}{16} \sum_{k=1}^{\infty} \lim _{N \rightarrow \infty} \sum_{|m| \leqq N}^{*} e^{-2 \pi i \nu m^{\prime} \mid k} \sum_{p=1}^{\infty} \frac{1}{p !}\left(\frac{2 \pi i \nu}{k(k \tau / j-m)}\right)^{p} \\
= & A+\frac{1}{16} \sum_{k=1}^{\infty} \lim _{N \rightarrow \infty} \sum_{|m| \leqq N}^{*} e^{-2 \pi i \nu m^{\prime} \mid k} \frac{2 \pi i \nu}{k(k \tau / j-m)} \\
& +\frac{1}{16} \sum_{k=1}^{\infty} \lim _{N \rightarrow \infty} \sum_{|m| \leqq N}^{*} e^{-2 \pi i \nu m^{\prime} \mid k} \sum_{p=2}^{\infty} \frac{1}{p !}\left(\frac{2 \pi i \nu}{k(k \tau / j-m)}\right)^{p} .
\end{aligned}
$$

The separation into two sums is justified since the first is convergent by Lemma (2.01) and the second is an absolutely convergent triple sum. 
It follows that the second sum can be rearranged in any fashion. Making use of this fact and noting that the restrictions $a<0, b<0, d>j c>0$ make it possible to apply Lemma (2.01), with $r=0$ and $a, b, c, d$ replaced by $a, b / j, j c, d$ to the first sum, we obtain

$$
\begin{aligned}
& \lambda_{\nu}(j ; \tau)=A+\frac{1}{16} \lim _{K \rightarrow \infty}\left\{\sum_{k=1}^{[K(a t-j c)]} \sum_{|m-b k / j a| \leqq K / a}^{*} \frac{e^{-2 \pi i \nu m^{\prime} / k}}{k(k \tau / j-m)} \cdot 2 \pi i \nu\right. \\
& \left.+\sum_{k=[K(a t-j c)]+1}^{[K(a t+j c)]} \sum_{(a k-K t) / j c \leqq m \leqq b k / j a+K / a}^{*} \frac{e^{-2 \pi i \nu m^{\prime} / k}}{k(k \tau / j-m)} \cdot 2 \pi i \nu\right\} \\
& +\frac{1}{16} \lim _{K \rightarrow \infty}\left\{\sum_{k=1}^{[K(a t-j c)]} \sum_{|m-b k| j a \mid \leqq K / a}^{*} e^{-2 \pi i \nu m / \mid k} \sum_{p=2}^{\infty} \frac{1}{p !}\left(\frac{2 \pi i \nu}{k(k \tau / j-m)}\right)^{p}\right.
\end{aligned}
$$

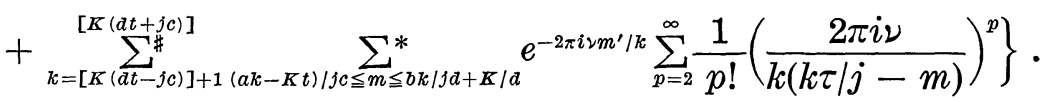

Therefore,

$$
\begin{aligned}
\lambda_{\nu}(j ; \tau)= & A+\frac{1}{16} \lim _{K \rightarrow \infty}\left\{\sum_{k=1}^{[K(a t-j c)]} \sum_{|m-b k / j a| \leqq K / a}^{*} e^{-2 \pi i \nu m^{\prime} / k}\left(\exp \left[\frac{2 \pi i \nu}{k(k \tau / j-m)}\right]-1\right)\right. \\
& \left.+\sum_{k=[K(a t-j c)]+1}^{[K(a t+j c)]} \sum_{(a k-K t) / j c \leqq m \leqq b k / J a+K / a}^{*} e^{-2 \pi i \nu m^{\prime} / k}\left(\exp \left[\frac{2 \pi i \nu}{k(k \tau / j-m)}\right]-1\right)\right\} .
\end{aligned}
$$

Now, let

$$
\begin{aligned}
S_{K}(\tau)= & \sum_{k=1}^{[K(a t-j c)]} \sum_{|m-b k / j a| \leqq K / a}^{*} e^{-2 \pi i \nu m^{\prime} / k} \exp \left[\frac{2 \pi i \nu}{k(k \tau / j-m)}\right] \\
& +\sum_{k=[K(a t-j c)]+1(a k-K t) / j c \leqq m \leqq b k / j a+K / a}^{[K(a t+j c)]} e^{-2 \pi i v m^{\prime} / k} \exp \left[\frac{2 \pi i \nu}{k(k \tau / j-m)}\right] .
\end{aligned}
$$

A little computing shows that

$$
\begin{aligned}
S_{K}(\tau)= & \sum_{k=1}^{[K(a t-j c)]} \sum_{|m-b k| j a] \leqq K / a}^{*} \exp \left[2 \pi i \nu \frac{-k^{\prime}-m^{\prime} \tau / j}{k \tau / j-m}\right] \\
& +\sum_{k=\left[K \left(\sum_{(a t-j c)]+1}^{\#}(a k-K t) \mid j c \leqq m \leqq b k / j a+K / a\right.\right.}^{[K(a t+j c)]} \exp \left[2 \pi i \nu \frac{-k^{\prime}-m^{\prime} \tau / j}{k \tau / j-m}\right],
\end{aligned}
$$

where $-k^{\prime}=\left(m m^{\prime}+1\right) / k$. We see that $k k^{\prime}+m m^{\prime}+1=0$, so $k k^{\prime} \equiv$ $-1(\bmod m)$. Now given the relatively prime pair $k, m$, the pair $k^{\prime}, m^{\prime}$ is not uniquely determined. In fact, $m^{\prime}$ can be replaced by $m^{\prime}+q k$, where $q$ is any integer. Then $k$ must be replaced by $k^{\prime}-q m$. The corresponding term in $S_{K}(\tau)$ is replaced by

$$
\begin{aligned}
& \exp \left[2 \pi i \nu \frac{-k^{\prime}+q m-\left(m^{\prime}+q k\right) \tau / j}{k \tau / j-m}\right]=\exp \left[2 \pi i \nu\left(\frac{-k^{\prime}-m^{\prime} \tau / j}{k \tau / j-m}-q\right)\right] \\
& =\exp \left[2 \pi i \nu\left(\frac{-k^{\prime}-m^{\prime} \tau / j}{k \tau / j-m}\right)\right] \text {, }
\end{aligned}
$$


so that $S_{K}(\tau)$ is unaffected by the ambiguity in the choice of $k^{\prime}$ and $m^{\prime}$.

Recall that in $S_{K}(\tau)$ we are summing over the lattice points of the trapezoid bounded by the lines $k=0, m=b k / j d-K / d, m=b k / j d+K / d$, $m=(a k-K t) / j c$. Now, if the pair $k, m$ is replaced by $-k,-m$, the pair $k^{\prime}, m^{\prime}$ is replaced by $-k^{\prime},-m^{\prime}$, and the corresponding term in $S_{K}(\tau)$ is unchanged. Therefore, if we extend our region of summation in $S_{K}(\tau)$ by reflecting the trapezoid through the origin, $S_{K}(\tau)$ is multiplied by 2 . The new region of summation is the parallelogram, $\mathscr{P}(K)$, bounded by the four lines $m=b k / j d \pm K / d, m=(a k \pm K t) / j c$. Therefore,

$$
S_{K}(\tau)=\frac{1}{2} \sum_{(k, m) € \mathscr{P}(K)}^{\#} \sum^{*} \exp \left[2 \pi i \nu \frac{-k^{\prime}-m^{\prime} \tau / j}{k \tau / j-m}\right] .
$$

It follows from this that

$$
S_{K}\left(\frac{a \tau+b}{c \tau+d}\right)=\frac{1}{2} \sum_{(k, m) \in \mathscr{P}(K)}^{\#} \sum^{*} \exp \left[2 \pi i \nu \frac{-\left(d k^{\prime}+b m^{\prime} / j\right)-(\tau / j)\left(j c k^{\prime}+a m^{\prime}\right)}{(\tau / j)(a k-j c m)-(m d-b k / j)}\right] .
$$

If the transformation $l=a k-j c m, n=-b k / j+m d$ is performed, the parallelogram $\mathscr{P}(K)$ in the $k-m$ plane is mapped onto the rectangle defined by $|l| \leqq t K,|n| \leqq K$ in the $l-n$ plane. Furthermore, since $a \equiv d \equiv 1(\bmod j), b \equiv c \equiv 0(\bmod j)$, and $a d-b c=1$, there is a one-to-one correspondence set up between the set of all lattice points $(k, m)$ in $\mathscr{P}(K)$ and the set of all lattice points $(l, n)$ of the rectangle $|l| \leqq t K,|n| \leqq K$. Also, a little computing shows that $(a k-j c m)\left(d k^{\prime}+b m^{\prime} \mid j\right)+$ $(m d-b k / j)\left(j c k^{\prime}+a m^{\prime}\right)+1=k k^{\prime}+m m^{\prime}+1=0$. Therefore we can put $l^{\prime}=d k^{\prime}+b m^{\prime} \mid j, n^{\prime}=j c k^{\prime}+a m^{\prime}$, and we finally obtain

$$
\begin{aligned}
S_{K}\left(\frac{a \tau+b}{c \tau+d}\right) & =\frac{1}{2} \sum_{|l| \leqq t K} \sum_{|n| \leqq K}^{*} \exp \left[2 \pi i \nu \frac{-l^{\prime}-n^{\prime} \tau \mid j}{l \tau \mid j-n}\right] \\
& =\sum_{i=1}^{[t K]} \sum_{|n| \leqq K}^{*} \exp \left[2 \pi i \nu \frac{-l^{\prime}-n^{\prime} \tau \mid j}{l \tau \mid j-n}\right] .
\end{aligned}
$$

Therefore, it follows from (4.05) that

$$
\begin{aligned}
& \lambda_{\nu}\left(j ; \frac{a \tau+b}{c \tau+d}\right) \\
& =A+\frac{1}{16} \lim _{K \rightarrow \infty}\left\{S_{K}\left(\frac{a \tau+b}{c \tau+d}\right)-\left[\sum_{k=1}^{[K(a t-j c)]} \sum_{|m-b k / j a| \leqq K / a}^{*} e^{-2 \pi i \nu m^{\prime} / k}\right.\right. \\
& \left.-\sum_{k=[K(a t-j c)]+1}^{[K(a t+j c)]} \sum_{(a k-K t) / j c \leqq m \leqq b k / j a+K / a}^{*} e^{-2 \pi i \nu m^{\prime} / k}\right\} \\
& =A+\frac{1}{16} \lim _{K \rightarrow \infty}\left\{\sum_{l=1}^{[t K]} \sum_{|n| \leqq K}^{*} \exp \left[2 \pi i \nu^{\prime} \frac{-l^{\prime}-n^{\prime} \tau / j}{l \tau \mid j-n}\right]\right. \\
& -\sum_{k=1}^{[K(a t-j c)]} \sum_{|m-b k / j a| \leqq K / a}^{*} e^{-2 \pi i \nu m^{\prime} / k}
\end{aligned}
$$




$$
\left.-\sum_{k=[K(a t-j c)]+1}^{[K(a t+j c)]} \sum_{(a k-K t) / j c \leqq m \leqq b k / j a+K / a}^{*} e^{-2 \pi i \nu m^{\prime} / k}\right\} .
$$

We now return to (4.01) and apply Lemma (2.03) with $r=0, \rho=t$. Proceeding in the same way as in the proof of (4.05), we find that (4.09) $\quad \lambda_{\nu}(j ; \tau)$

$$
\begin{aligned}
& =A+\frac{1}{16} \lim _{K \rightarrow \infty} \sum_{k=1}^{[t K]} \sum_{|m| \leqq K}^{*} e^{-2 \pi i \nu m^{\prime} \mid k}\left(\exp \left[\frac{2 \pi i \nu}{k(k \tau / j-m)}\right]-1\right) \\
& =A+\frac{1}{16} \lim _{K \rightarrow \infty}\left\{\sum_{k=1}^{[t K]} \sum_{|m| \leqq K}^{*} \exp \left[2 \pi i \nu \frac{-k^{\prime}-m^{\prime} \tau \mid j}{k \tau \mid j-m}\right]-\sum_{k=1}^{[t K]} \sum_{|m| \leqq K}^{*} e^{-2 \pi i \nu m^{\prime} \mid k}\right\} .
\end{aligned}
$$

Upon comparing (4.08) and (4.09), we conclude that

$$
\begin{aligned}
\lambda_{\nu}(j ; & \left.\frac{a \tau+b}{c \tau+d}\right)-\lambda_{\nu}(j ; \tau) \\
= & \frac{1}{16} \lim _{k \rightarrow \infty}\left\{\sum_{k=1}^{[t K]} \sum_{|m| \leqq K}^{*} e^{-2 \pi i \nu m^{\prime} / k}-\sum_{k=1}^{[K(a t-j c)]} \sum_{|m-b k / J d| \leqq K / a}^{*} e^{-2 \pi \imath \nu m^{\prime} / k}\right. \\
& \left.-\sum_{k=[K(a t-j c)]+1}^{[K(a t+j c)]} \sum_{(a k-K t) / j c \leqq m \leqq b k / j a+K / a}^{\#} e^{-2 \pi i \nu m^{\prime} / k}\right\} \equiv \omega_{\nu}(j ; c, d) .
\end{aligned}
$$

We have proved the required transformation properties when $\tau$ is a pure imaginary number. But $\lambda_{\nu}(j ; \tau)$ is regular for $\mathscr{I}(\tau)>0$, and therefore, by analytic continuation, (4.10) holds for $\mathscr{I}(\tau)>0$, and the proof of the theorem is complete.

There are other transformation properties of the $\lambda_{\nu}(j ; \tau)$ for special values of $\nu$. These can be summarized in the following.

ThEOREM (4.11). (a) If $\nu$ is a multiple of $j$, then for $\mathscr{I}(\tau)>0$,

$$
\lambda_{\nu}(j ;-1 / \tau)=\lambda_{\nu}(j ; \tau) .
$$

(b) If $j$ is even and $\nu$ is an odd multiple of $j / 2$, then for $\mathscr{I}(\tau)>0$,

$$
\lambda_{\nu}(j ;-1 / \tau)=\sigma_{\nu}(j)-\lambda_{\nu}(j ; \tau),
$$

where $\sigma_{\nu}(j)$ does not depend on $\tau$.

Proof. We again begin by assuming that $\tau$ is a pure imaginary number. Returning to (4.01), applying Lemma (2.03) with $r=0, \rho=j$, and proceeding as in the proof of (4.05), we obtain

$$
\lambda_{\nu}(j ; \tau)=A+\frac{1}{16} \lim _{K \rightarrow \infty} \sum_{k=1}^{j K} \sum_{|m| \leqq K}^{*} e^{-2 \pi i \nu m^{\prime} / k}\left(\exp \left[\frac{2 \pi i \nu}{k(k \tau / j-m)}\right]-1\right) .
$$

This time, put

$$
S_{K}(\tau)=\sum_{k=1}^{j k} \sum_{|m| \leqq K}^{*} e^{-2 \pi i \nu m^{\prime} / k} \exp \left[\frac{2 \pi i \nu}{k(k \tau / j-m)}\right]
$$




$$
\begin{aligned}
= & \sum_{k=1}^{j K} \sum_{m=1}^{K} * \exp \left[2 \pi i \nu \frac{-k^{\prime}-m^{\prime} \tau / j}{k \tau / j-m}\right] \\
& +\sum_{k=1}^{j K} \sum_{m=1}^{K} * \exp \left[2 \pi i \nu \frac{-k^{\prime}+m^{\prime} \tau / j}{k \tau / j+m}\right],
\end{aligned}
$$

where we have separated the terms for $m<0$ and $m>0$.

It follows that

$$
\begin{aligned}
S_{K}(-1 / \tau)= & \sum_{k=1}^{j K} \sum_{m=1}^{K} * \exp \left[2 \pi i \nu \frac{-k^{\prime} \tau+m^{\prime} \mid j}{-k / j-m \tau}\right] \\
& +\sum_{k=1}^{j K} \sum_{m=1}^{K} * \exp \left[2 \pi i \nu \frac{-k^{\prime} \tau-m^{\prime} \mid j}{-k / j+m \tau}\right] .
\end{aligned}
$$

Put $l=k / j$ and $n=j m$; it follows from $(k, m)=1, k \equiv j\left(\bmod j^{2}\right)$, and $m \equiv 1(\bmod j)$ that $(l, n)=1, l \equiv 1(\bmod j)$, and $n \equiv j\left(\bmod j^{2}\right)$. Also, we may put $l^{\prime}=j k^{\prime}-m, n^{\prime}=\left(k / j+m^{\prime}\right) / j$. For $m m^{\prime} \equiv-1(\bmod k), m \equiv 1(\bmod j)$, and $j \mid k$ together imply that $m^{\prime} \equiv-1(\bmod j)$. Using the fact that $k / j \equiv 1(\bmod j)$, we find that $k / j+m^{\prime} \equiv 0(\bmod j)$ and $n^{\prime}$, as defined above, is an integer. Furthermore, $l l^{\prime}+n n^{\prime}+1=k k^{\prime}+m m^{\prime}+1=0$. With the above definition of $l^{\prime}$ and $n^{\prime}$, we have $k^{\prime}=\left(l^{\prime}+n / j\right) / j$ and $m^{\prime}=j n^{\prime}-l$. Now, (4.16) becomes

$$
\begin{aligned}
S_{K}(-1 / \tau)= & \sum_{n=1}^{j K} \sum_{l=1}^{K} * \exp \left[2 \pi i \nu \frac{-\left(l^{\prime}+n / j\right) \tau / j+\left(j n^{\prime}-l\right) / j}{-l-n \tau / j}\right] \\
& +\sum_{n=1}^{j K} \sum_{l=1}^{K} * \exp \left[2 \pi i \nu \frac{-\left(l^{\prime}+n / j\right) \tau / j-\left(j n^{\prime}-l\right) / j}{-l+n \tau / j}\right] \\
= & \sum_{n=1}^{j K} \sum_{l=1}^{K} * \exp \left[2 \pi i \nu\left(\frac{-n^{\prime}+l^{\prime} \tau / j}{n \tau / j+l}+1 / j\right)\right] \\
& +\sum_{n=1}^{j K} \sum_{l=1}^{K} * \exp \left[2 \pi i \nu\left(\frac{-n^{\prime}-l^{\prime} \tau / j}{n \tau / j-l}-1 / j\right)\right] .
\end{aligned}
$$

We see from (4.14) and the definition of $S_{K}(\tau)$ that

$$
\lambda_{\nu}(j ; \tau)=A+\frac{1}{16} \lim _{K \rightarrow \infty}\left\{S_{K}(\tau)-\sum_{k=1}^{j K} \sum_{\mid m \leqq K}^{*} e^{-2 \pi i \nu m^{\prime} / k}\right\} .
$$

Now, if $\nu$ is a multiple of $j$, a comparison of (4.15) and (4.17) shows that $S_{K}(-1 / \tau)=S_{K}(\tau)$ and therefore (4.12) follows. This is part (a) of the theorem. In part $(\mathrm{b}), S_{K}(-1 / \tau)=-S_{K}(\tau)$, and therefore,

$$
\lambda_{\nu}(j ;-1 / \tau)+\lambda_{\nu}(j ; \tau)=2 A-\frac{1}{8} \lim _{K \rightarrow \infty} \sum_{k=1}^{j K} \sum_{|m| \leqq K}^{*} e^{-2 \pi i \nu m^{\prime} \mid k} \equiv \sigma_{\nu}(j) .
$$

This is part (b) of the theorem. Here again the theorem has been proved for $\tau$ a pure imaginary number, but as before we extend our results by analytic continuation to all $\tau$ such that $\mathscr{F}(\tau)>0$. 
5. Construction of functions for $G(j)$. In order to construct functions which are invariant under the group $G(j)$, we make use of Theorem (4.02) and the fact that $G(j)$ is finitely generated. Let $T_{l}, l=1, \cdots, q(j)$, be a set of generators for $G(j)$. Then by Theorem (4.02), we have

$$
\lambda_{\nu}\left(j ; T_{l}(\tau)\right)-\lambda_{\nu}(j ; \tau)=c_{l, \nu}(j),
$$$$
l=1, \cdots, q(j),
$$

for any integer $\nu \geqq 1$.

Let $1 \leqq \nu_{1}<\nu_{2}<\cdots<\nu_{m}$ be integers and consider the function defined by

$$
F(\tau)=b_{1} \lambda_{\nu_{1}}(j ; \tau)+\cdots+b_{m} \lambda_{\nu_{m}}(j ; \tau) .
$$

Then $F(\tau)$ satisfies the functional equations

$$
F\left(T_{l}(\tau)\right)-F(\tau)=b_{1} c_{l, \nu_{1}}(j)+\cdots+b_{m} c_{l, \nu_{m}}(j), \quad l=1, \cdots, q(j) .
$$

Let $m \geqq q(j)+1$ and consider the homogeneous linear system in the $m$ unknowns $b_{1}, \cdots, b_{m}$

$$
b_{1} c_{l, \nu_{1}}(j)+\cdots+b_{m} c_{l, \nu_{m}}(j)=0, \quad l=1, \cdots, q(j) .
$$

This has $m-q(j)$ linearly independent solutions $\left(b_{1}, \cdots, b_{m}\right)$. With $b_{1}, \cdots, b_{m}$ chosen to satisfy (5.04), put

$$
\mathscr{L}\left(j ; b_{1}, \cdots, b_{m} ; \nu_{1}, \cdots, \nu_{m} ; \tau\right)=b_{1} \lambda_{\nu_{1}}(j ; \tau)+\cdots+b_{m} \lambda_{\nu_{m}}(j ; \tau) .
$$

It follows from (5.03) and (5.04) that $\mathscr{L}\left(j ; b_{1}, \cdots, b_{m} ; \nu_{1}, \cdots, \nu_{m} ; T_{l}(\tau)\right)=$ $\mathscr{L}\left(j ; b_{1}, \cdots, b_{m} ; \nu_{1}, \cdots, \nu_{m} ; \tau\right)$ for $l=1, \cdots, q(j)$ and therefore, since the $T_{\imath}$ generate $G(j)$, we have

(5.06) $\mathscr{L}\left(j ; b_{1}, \cdots, b_{m} ; \nu_{1}, \cdots, \nu_{m} ; T(\tau)\right)=\mathscr{L}\left(j ; b_{1}, \cdots, b_{m} ; \nu_{1}, \cdots, \nu_{m} ; \tau\right)$, for all $T$ in $G(j)$.

In order to show that the function $\mathscr{L}$ defined by (5.05) cannot reduce to a constant we prove

Lemma (5.07). Let $d_{n}$ be the $n$th Fourier coefficient of the function L. Then

$$
d_{n} \sim\left(b_{m} / 16\right) \nu_{m}^{1 / 4} n^{-3 / 4}(2 j)^{-1 / 2} e^{-2 \pi i\left(n-\nu_{m}\right) / 9} \exp \left[4 \pi\left(n \nu_{m}\right)^{1 / 2} / j\right] .
$$

Proof. We see immediately from (5.05) that $d_{n}=\sum_{i=1}^{m} b_{i} a_{n}\left(\nu_{i}, j\right)$, with $a_{n}\left(\nu_{i}, j\right)$ defined as in (3.01). The lemma now is direct consequence of Lemma (3.02a)

In particular, (5.08) implies that $\mathscr{L}$ is not a constant.

6. Construction of forms for $G(j)$. Let $r$ be any positive even 
integer. We define the function

$$
\begin{aligned}
& \lambda_{\nu}(j ; \tau, r)=\sum_{n=1}^{\infty} a_{n}(\nu, j, r) e^{2 \pi i n \tau / j} \\
& a_{n}(\nu, j, r)=\left\{(-1)^{r / 2} \pi / 8\right\} \sum_{k=1}^{\infty} k^{-1} A_{k, \nu}(n) \cdot(\nu / n)^{(r+1) / 2} I_{r+1}\left(4 \pi(n \nu)^{1 / 2} / k\right),
\end{aligned}
$$

where $A_{k, \nu}(n)$ is defined as in (3.01) and $I_{r+1}$ is again a Bessel function of the first kind. It should be noted that if we put $r=0$ in (6.01) we obtain the function $\lambda_{\nu}(j ; \tau)$ defined by (3.01).

The computations of $\S \S 3$ and 4, using Lemmas (2.01) and (2.03), with $r>0$, yield the following two theorems.

THEOREM $(6.02)^{2}$. The function $\lambda_{\nu}(j ; \tau, r)$ satisfies the transformation equations

$$
\begin{aligned}
(c \tau+d)^{r} \lambda_{\nu}\left(j ; T_{a, b, c, a}(\tau), r\right) & \equiv(c \tau+d)^{r} \lambda_{\nu}\left(j ; \frac{a \tau+b}{c \tau+d}, r\right) \\
& =\lambda_{\nu}(j ; \tau, r)+p_{\nu}(j ; \tau, r ; c, d),
\end{aligned}
$$

for all $T_{a, b, c, a}$ in $G(j)$ and $\mathscr{I}(\tau)>0$, where $p_{\nu}(j ; \tau, r ; c, d)$ is a polynomial in $\tau$ of degree at most $r$.

Theorem (6.04). (a) If $\nu$ is a multiple of $j$, then for $\mathscr{I}(\tau)>0$,

$$
\tau^{r} \lambda_{\nu}(j ;-1 / \tau, r)=\lambda_{\nu}(j ; \tau, r)+p_{\nu, 1}(j ; \tau, r),
$$

where $p_{\nu, 1}(j ; \tau, r)$ is a polynomial in $\tau$ of degree at most $r$.

(b) If $j$ is even and $\nu$ is an odd multiple of $j / 2$, then for $\mathscr{I}(\tau)>0$,

$$
\tau^{r} \lambda_{\nu}(j ;-1 / \tau, r)=p_{\nu, 2}(j ; \tau, r)-\lambda_{\nu}(j ; \tau, r),
$$

where $p_{\nu, 2}(j ; \tau, r)$ is a polynomial in $\tau$ of degree at most $r$.

Now, in order to construct forms of dimension $r$ for $G(j)$, we make use of Theorem (6.02) and proceed as in $\S 5$. We take a linear combination of the $\lambda_{\nu}(j ; \tau, r)$ in such a way that the resulting linear combination of polynominals occurring in the transformation equation connected with $T_{l}, l=1, \cdots, q(j)$, vanishes identically. In this case $m$, the number of $\lambda_{\nu}(j ; \tau, r)$ in the linear combination, must be such that $m \geqq$ $(r+1) \cdot q(j)+1$.

A simple generalization of Lemma (5.07), to cover the present case, shows that the forms constructed in this way are not identically zero.

7. Conclusion. Other functions of the type dealt with in this paper can be constructed by generalizing the congruence conditions on $k$ and $h$ in (3.01) and (6.01). Let $n_{1}$ and $n_{2}$ be any integers. If, in

${ }^{2}$ See correction at end of paper. 
(3.01), we impose the new congruence conditions $k \equiv n, j\left(\bmod j^{2}\right), h \equiv$ $n_{2}(\bmod j)$, we obtain new functions which satisfy (4.03), and which, therefore, can be used to construct functions which are invariant under $G(j)$.

If $\left(n_{2}, j\right)>1$, the sum defining $A_{k, \nu}(n)$ is empty and so each Fourier coefficient is zero. Also the case $n_{1} \equiv 0(\bmod j), n_{2} \equiv 1(\bmod j)$ is unique and will receive separate treatment in another publication. The distinctive feature here is the fact that, in order to construct functions satisfying (4.03), we must introduce a pole term at $i \infty$. This situation occurs, for example, in the Fourier expansion of $\mu(\tau)$, the reciprocal of $\lambda(\tau)$ (see [6]).

Making the additional assumptions $n_{1}=n_{2}, n_{1}^{2} \equiv 1(\bmod j)$ in (3.01), we obtain functions for which we can prove Theorem (4.11).

Correspondingly, if we impose the conditions $k \equiv n_{1}, j\left(\bmod j^{2}\right)$, $h \equiv n_{2}(\bmod j)$ in $(6.01)$, we obtain functions satisfying (6.03), and making the further assumptions, $n_{1}=n_{2}, n_{1}^{2} \equiv 1(\bmod j)$, we obtain functions for which Theorem (6.04) holds.

It should be noted that all of our functions vanish at the parabolic cusp at infinity. As the referee has pointed out, it is of interest to consider the behavior of these functions at the other parabolic cusps of $G(j)$. This question will be treated at a later time.

Correction to "Construction of a Class of Modular Functions and Forms". As it stands the proof of Theorem (4.02) is incorrect. The difficulty arises in the paragraph immediately preceding (4.06), where we extend the region of summation in $S_{K}(\tau)$. In the original expression. for $S_{K}(\tau)$ we are summing over the points $(k, m)$ of a certain trapezoid subject to the additional restrictions $(m, k)=1, k \equiv j\left(\bmod j^{2}\right), m=1(\bmod j)$. In order to extend the region of summation to the parallelogram $\mathscr{P}(K)$, we reflect this trapezoid through the origin. That is, when $(k, m)$ appears in the summation, we also include the point $(-k,-m)$. The trouble is, that when $j \geqq 3,(-k,-m)$ does not satisfy the proper congruence conditions, but rather the new conditions $-k \equiv-j\left(\bmod j^{2}\right),-m \equiv-1(\bmod j)$, or equivalently, $-k \equiv j^{2}-j\left(\bmod j^{2}\right),-m \equiv j-1(\bmod j)$. Hence the expression (4.06) for $S_{K}(\tau)$ is incorrect, when $j \geqq 3$. For $j=2$, of course, this difficulty does not arise.

The situation can be readily rectified if we go back to (3.01) and modify the definition of the function $\lambda_{\nu}(j ; \tau)$. Put $b_{n}^{+}(\nu, j)=a_{n}(\nu, j)$, with $a_{n}(\nu, j)$ as in (3.01) and define $b_{n}^{-}(\nu, j)$ to be the same as $a_{n}(\nu, j)$, except that the congruence condition on $k$ is changed to $k \equiv j^{2}-j\left(\bmod j^{2}\right)$. and the congruence condition on $h$ is changed to $h \equiv j-1(\bmod j)$. We now define $\lambda_{\nu}(j ; \tau)$ by

$$
\lambda_{\nu}(j ; \tau)=\sum_{n=1}^{\infty} b_{n}(\nu, j) e^{2 \pi i n \tau / j},
$$


where

$$
b_{n}(\nu, j)=\frac{1}{2}\left[b_{n}^{+}(\nu, j)+b_{n}^{-}(\nu, j)\right] .
$$

when $j=2, b_{n}^{+}(\nu, j)=b_{n}^{-}(\nu, j)=b_{n}(\nu, j)=a_{n}(\nu, j)$, and no change has been made.

With this new definition of $\lambda_{\nu}(j ; \tau)(4.06)$ becomes

$$
S_{K}(\tau)=\frac{1}{2} \sum \sum \exp \left[2 \pi i \nu \frac{-k^{\prime}-m^{\prime} \tau / j}{k \tau / j-m}\right]
$$

where the summation is over all points of $\mathscr{P}(K)$ such that $(m, k)=1$. and $e i t h e r k \equiv j\left(\bmod j^{2}\right), m \equiv 1(\bmod j)$ or $k \equiv j^{2}-j\left(\bmod j^{2}\right), m \equiv j-1(\bmod j)$ The remainder of the proof now carries through.

The same remark is necessary in connection with Theorem (6.02). That is, Theorem (6.02) is incorrect as it stands, but if we modify the function $\lambda_{\nu}(j ; \tau, r)$ in the same way as we modified $\lambda_{\nu}(j ; \tau)$, the proof goes through.

We should point out that Theorems (4.11) and (6.04) are correct as they are, but in addition Theorem (4.11) is true for the modified $\lambda_{\nu}(j ; \tau)$ and Theorem (6.04) is true for the modified $\lambda_{\nu}(j ; \tau, r)$.

Similar modifications have to be made in the definition of the functions mentioned in $\$ 7$.

\section{BIBLIOGRAPHY}

1. M. I. Knopp, Fourier series of automorphic forms of nonnegative dimension, Illinois J. Math. to appear.

2. J. Lehner, On modular forms of negative dimension, Michigan Math. J. 6 (1959), 71-88.

3. R. Lipschitz, Untersuchung der eigenschaften einer gattung von unendlichen Reihen, Journal für Math. 105 (1889), 127-156.

4. H.Rademacher, The Fourier series and the functional equation of the absolute modular invariant $J(\tau)$, Amer. J. Math. 61 (1939), 237-248.

5. H. Salié, Zur abschätzung der Fourierkoeffizienten ganzer modulformen, Math. Zeit. 36 (1933), 263-278.

6. W. H. Simons, The Fourier coefficients of the modular function $\lambda(\tau)$, Canadian J. Math. 4 (1952), 67-80.

7. G. N. Watson, Theory of Bessel Functions, Cambridge, Cambridge University Press, 1945.

The Institute For AdVANCEd Study

Princeton, New Jersey 



\section{PACIFIC JOURNAL OF MATHEMATICS}

\section{EDITORS}

Ralph S. Phillips

Stanford University

Stanford, California

F. H. BrownelL

University of Washington

Seattle 5 , Washington
A. L. Whiteman

University of Southern California Los Angeles 7. California

L. J. PAIGE

University of California

Los Angeles 24, California

ASSOCIATE EDITORS
E. F. BECKENBACH
D. DERRY
H. L. ROYDEN
E. G. STRAUS
T. M. CHERRY
M. OHTSUKA
E. SPANIER
F. WOLF

\section{SUPPORTING INSTITUTIONS}

UNIVERSITY OF BRITISH COLUMBIA

CALIFORNIA INSTITUTE OF TECHNOLOGY

UNIVERSITY OF CALIFORNIA

MONTANA STATE UNIVERSITY

UNIVERSITY OF NEVADA

NEW MEXICO STATE UNIVERSITY

OREGON STATE COLLEGE

UNIVERSITY OF OREGON

OSAKA UNIVERSITY

UNIVERSITY OF SOUTHERN CALIFORNIA
STANFORD UNIVERSITY

UNIVERSITY OF TOKYO

UNIVERSITY OF UTAH

WASHINGTON STATE COLLEGE,

UNIVERSITY OF WASHINGTON

AMERICAN MATHEMATICAL SOCIETY

CALIFORNIA RESEARCH CORPORATION

HUGHES AIRCRAFT COMPANY

SPACE TECHNOLOGY LABORATORIES

NAVAL ORDNANCE TEST STATION

Printed in Japan by International Academic Printing Co., Ltd., Tokyo, Japan

Reprinted 1966 in the United States of America 


\section{Pacific Journal of Mathematics}

\section{Vol. 11, No. $1 \quad$ November, 1961}

A. A. Albert, Generalized twisted fields ............................ 1

Richard Arens, Operational calculus of linear relations ................... 9

John Herbert Barrett, Disconjugacy of a self-adjoint differential equation of the fourth order ....................................... 25

Paul Richard Beesack, Hardy's inequality and its extensions ............... 39

Julius Rubin Blum and David Lee Hanson, On invariant probability measures.

II .............................................

Robert Allen Bonic, Symmetry in group algebras of discrete groups.......... 73

R. Creighton Buck, Multiplication operators ...................... 95

Jack Gary Ceder, Some generalizations of metric spaces ................. 105

Meyer Dwass, Random crossings of cumulative distribution functions ......... 127

Albert Edrei, Wolfgang H. J. Fuchs and Simon Hellerstein, Radial distribution and

deficiencies of the values of a meromorphic function ............... 135

William Cassidy Fox, Harmonic functions with arbitrary local singularities ..... 153

Theodore Thomas Frankel, Manifolds with positive curvature ............... 165

Avner Friedman, A strong maximum principle for weakly subparabolic

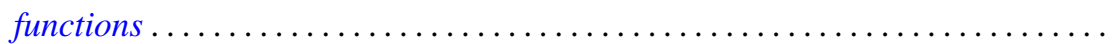

Watson Bryan Fulks and J. O. Sather, Asymptotics. II. Laplace's method for

multiple integrals ......................................

Adriano Mario Garsia and Eugene Richard Rodemich, An embedding of Riemann

surfaces of genus one ..................................... 193

Irving Leonard Glicksberg, Weak compactness and separate continuity......... 205

Branko Grünbaum, On a conjecture of H. Hadwiger .................. 215

Frank J. Hahn, On the action of a locally compact group on $E_{n} \ldots \ldots \ldots \ldots \ldots . . \ldots 221$

Magnus R. Hestenes, Relative hermitian matrices ..................... 225

G. K. Kalisch, On similarity invariants of certain operators in $L_{p} \ldots \ldots \ldots \ldots .247$

Yitzhak Katznelson and Walter Rudin, The Stone-Weierstrass property in Banach

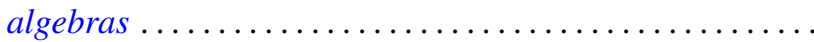

Samir A. Khabbaz, The subgroups of a divisible group $G$ which can be represented as intersections of divisible subgroups of $G \ldots \ldots \ldots \ldots \ldots \ldots \ldots \ldots \ldots . \ldots \ldots 7$

Marvin Isadore Knopp, Construction of a class of modular functions and forms .......................................... 275

Charles Alan McCarthy, Commuting Boolean algebras of projections .......... 295

T. M. MacRobert, Transformations of series of E-functions ................ 309

Heinz Renggli, An inequality for logarithmic capacities ................. 313

M. S. Robertson, Applications of the subordination principle to univalent functions .......................................... 315

David Sachs, Partition and modulated lattices ..................... 325

Frank S. Scalora, Abstract martingale convergence theorems ............... 347

Elbert A. Walker, Torsion endomorphic images of mixed Abelian groups ........ 375

Morgan Ward, The prime divisors of Fibonacci numbers................. 379

Charles R. B. Wright, On the nilpotency class of a group of exponent four....... 387 